\title{
ARCS DEFINED BY ONE-PARAMETER SEMIGROUPS OF OPERATORS
}

\author{
HUGO D. JUNGHENN AND C. T. TAAM ${ }^{1}$
}

\begin{abstract}
Let $T(t)(t \geqq 0)$ be a one-parameter semigroup of continuous linear operators in a locally convex reflexive linear topological space $X$ such that $T(c)$ is an isomorphism (into) for some $c>0$. It is proved that for any $x \in X, T(\cdot) x$ is of bounded variation on finite intervals if and only if $x$ is in the domain of the infinitesimal generator of $T(t)$. The result is interpreted geometrically in terms of arc-length.
\end{abstract}

1. Definitions and main results. Let $X$ be a locally convex sequentially complete (Hausdorff) linear topological space over the complex numbers, and let $X^{*}$ and $L(X)$ denote the dual space and the space of all continuous linear operators in $X$ respectively. A one-parameter semigroup is a family $\{T(t): t \geqq 0\} \subset L(X)$ with the following properties:

(1) $T(t) T(s)=T(t+s)$ for any $t, s \geqq 0$;

(2) $T(0)=I$, the identity operator;

(3) $\lim _{t \rightarrow s} T(t) x=T(s) x$ for any $s \geqq 0$ and any $x \in X$.

A one-parameter semigroup $\{T(t): t \geqq 0\} \subset L(X)$ is said to be locally equicontinuous if for any $0<s<\infty$ and any continuous seminorm $p$ on $X$ there exists a continuous seminorm $q$ on $X$ such that $p(T(t) x) \leqq q(x)$ for all $0 \leqq t \leqq s$ and all $x \in X$. We refer the reader to the paper by $\mathrm{T}$. Komura [2] for the basic properties of locally equicontinuous semigroups. In particular we note that a one-parameter semigroup is locally equicontinuous if the space $X$ is reflexive.

The infinitesimal generator $A$ of a one-parameter semigroup $\{T(t)$; $t \geqq 0\}$ is defined by

$$
A x=\lim _{h \downarrow 0}(T(h) x-x) / h
$$

whenever this limit exists. The domain of $A$, denoted $D(A)$, is always

Received by the editors April 14, 1973.

AMS (MOS) subject classifications (1970). Primary 46A05, 47D05; Secondary 46A25.

Key words and phrases. Semigroup of operators, reflexive, arc-length, bounded variation, absolute continuity, local equicontinuity.

${ }^{1}$ Research of second author was partially supported by the U.S. Army Research Office, Durham, under contract number DAWC-04-67-C-0056.

(c) American Mathematical Society 1974 
dense in $X$, and $A$ is a linear operator from $D(A)$ into $X$. Furthermore, if $\{T(t): t \geqq 0\}$ is locally equicontinuous, then $A$ is closed [2].

Let $x \in X, 0 \leqq a \leqq b<\infty$, and let $p$ be any continuous seminorm on $X$. We define the $p$-length $L(x ; a, b ; p)$ of the $\operatorname{arc}\{T(t) x: a \leqq t \leqq b\}$ by

$$
L(x ; a, b ; p)=\sup \sum_{j=1}^{n} p\left(T\left(t_{j}\right) x-T\left(t_{j-1}\right) x\right)
$$

where the supremum is taken over all finite sums determined by partition points $a=t_{0}<t_{1}<\cdots<t_{n}=b$. If $L(x ; a, b ; p)<\infty$ for every continuous seminorm $p$, we say that the function $T(\cdot) x:[0, \infty) \rightarrow X$ is of bounded variation on the interval $[a, b]$. Finally $T(\cdot) x$ is said to be absolutely continuous on $[a, b]$ if for every positive $\varepsilon$ and continuous seminorm $p$ on $X$ there exists a positive $\delta$ such that

$$
\sum_{j=1}^{n} p\left(T\left(b_{j}\right) x-T\left(a_{j}\right) x\right)<\varepsilon
$$

whenever $\left(a_{1}, b_{1}\right),\left(a_{2}, b_{2}\right), \cdots,\left(a_{n}, b_{n}\right)$ are nonoverlapping subintervals of $[a, b]$ with

$$
\sum_{j=1}^{n}\left(b_{j}-a_{j}\right)<\delta .
$$

We recall that an isomorphism of a locally convex space $X$ into itself is a continuous linear transformation on $X$ which is one-one and relatively open.

THEOREM. Let $X$ be a locally convex reflexive linear topological space and $\{T(t): t \geqq 0\} \subset L(X)$ a one-parameter semigroup with infinitesimal generator $A$. Suppose $T(c)$ is an isomorphism for some $c>0$. Then for each $x \in X$ the following are equivalent :

(i) $x \in D(A)$;

(ii) $T(\cdot) x$ is absolutely continuous on each finite subinterval $[a, b]$ of $[0, \infty)$

(iii) $T(\cdot) x$ is of bounded variation on each finite subinterval $[a, b]$ of $[0, \infty)$.

If any of the above statements holds then every arc $\{T(t) x: a \leqq t \leqq b\}$, where $0 \leqq a<b<\infty$, has finite p-length, and

$$
L(x ; a, b ; p)=\int_{a}^{b} p(A T(t) x) d t
$$

for each continuous seminorm $p$ on $X$.

Corollary. If $D(A) \neq X$ (for example if $X$ is an F-space and $A$ is not continuous) then for each interval $[a, b] \subset[0, \infty)$ there exist continuous 
seminorms $p$ on $X$ such that each nonempty open set contains points $x$ for which $L(x ; a, b ; p)$ is arbitrarily large (both finite and infinite).

Remarks. The implications (i) $\Rightarrow$ (ii) and (ii) $\Rightarrow$ (iii) of the Theorem are valid in the absence of reflexivity. That this is not the case for the implications (ii) $\Rightarrow$ (i) and (iii) $\Rightarrow$ (i) is demonstrated by the following example:

EXAmple 1. Let $X=L^{1}(-\infty, \infty)$ and let $T(t)$ be translation by $t$. $D(A)$ consists of those $x \in X$ which are absolutely continuous on finite intervals and have derivatives in $L^{1}$, and $A x=x^{\prime}$ [1]. If $x(t)$ is the characteristic function of the interval $[0,1]$, then $T(\cdot) x$ is absolutely continuous, but $x \notin D(A)$.

The next example shows that the implication (iii) $\Rightarrow$ (i) of the Theorem is not generally true if none of the operators $T(t)(t>0)$ is an isomorphism.

ExAmple 2. Let $X=L^{2}([0, \infty))$ and define $T(t)$ as in Example 1. Then $D(A)$ consists of all $x \in X$ which are absolutely continuous on finite intervals and have derivatives in $L^{2}$, and $A x=x^{\prime}$ [1]. Define $x \in X$ as follows:

$$
\begin{aligned}
x(s) & =s^{-1 / 4} & & \text { if } 0<s \leqq 1, \\
& =2-s & & \text { if } 1 \leqq s \leqq 2, \\
& =0 & & \text { otherwise. }
\end{aligned}
$$

It is easily verified that $T(t) x \in D(A)$ for all $t>0$, but $x \notin D(A)$. Since

$$
\|A T(t) x\| \leqq t^{-3 / 4}+1 \text { for all } t>0,
$$

it follows that for any partition $0 \leqq t_{0}<t_{1}<\cdots<t_{n}=b$ we have

$$
\begin{aligned}
\sum_{j=1}^{n}\left\|T\left(t_{j}\right) x-T\left(t_{j-1}\right) x\right\| & \leqq 2\|x\|+\sum_{j=2}^{n} \int_{t_{j-1}}^{t_{j}}\|A T(t) x\| d t \\
& \leqq 2\|x\|+b+4 b^{1 / 4} .
\end{aligned}
$$

Therefore $T(\cdot) x$ is of bounded variation on $[0, b]$ for every $0<b<\infty$. In fact, similar calculations show that $T(\cdot) x$ is absolutely continuous on $[a, \infty)$ for any $a>0$.

Our final example explicitly illustrates the phenomenon indicated in the Corollary.

EXAMPLE 3. Let $X=1^{p}, 1 \leqq p<\infty$, and define $T(t)$ by

$$
T(t) x=\left(e^{i t} x_{1}, e^{i 2 t} x_{2}, \cdots\right) ; \quad x=\left(x_{1}, x_{2}, \cdots\right) .
$$

Then $D(A)$ consists of all those $x=\left(x_{1}, x_{2}, \cdots\right) \in X$ such that

$$
\sum_{n=1}^{\infty}\left|n x_{n}\right|^{p}<\infty
$$


and $A x=\left(i x_{1}, 2 i x_{2}, \cdots\right)$. Choose any $z=\left(z_{1}, z_{2}, \cdots\right)$ not in $D(A)$, and for each pair of positive integers $k$ and $n$ define $y(k, n)=(0,0, \cdots$, $\left.0, z_{k}, z_{k+1}, \cdots, z_{k+n}, 0,0, \cdots\right)$. Then $\lim _{k \rightarrow \infty} y(k, n)=0$ uniformly in $n$, and for each $k$,

$$
L(y(k, n) ; a, b ;\|\cdot\|)=(b-a)\|A y(k, n)\| \rightarrow \infty
$$

as $n \rightarrow \infty$. Thus for fixed $0 \leqq a<b<\infty$ we may construct vectors $y$ in $D(A)$ with arbitrarily small norm and arbitrarily large arc-length.

2. Proof of Theorem. Assume statement (i) of the Theorem holds. It follows from the sequential completeness of $X$ and the continuity of $A T(\cdot) x$ on $[0, \infty)$ that the Riemann integral $\int_{a}^{b} A T(t) x d t$ exists in $X$ for any $0 \leqq a<b<\infty$. Moreover,

$$
T(b) x-T(a) x=\int_{a}^{b} A T(t) x d t
$$

[2]. Let $p$ be any continuous seminorm on $X$ and $0 \leqq a<b<\infty$. By the local equicontinuity of $\{T(t) ; t \geqq 0\}$ there exists a continuous seminorm $q$ on $X$ such that $p(A T(t) x) \leqq q(A T(a) x)$ for all $a \leqq t \leqq b$. Therefore if $\left(a_{1}, b_{1}\right),\left(a_{2}, b_{2}\right), \cdots,\left(a_{n}, b_{n}\right)$ are nonoverlapping intervals in $[a, b]$, we have from (1)

$$
\begin{aligned}
\sum_{j=1}^{n} p\left(T\left(b_{j}\right) x-T\left(a_{j}\right) x\right) & \leqq \sum_{j=1}^{n} \int_{a_{j}}^{b_{j}} p(A T(t) x) d t \\
& \leqq \sum_{j=1}^{n}\left(b_{j}-a_{j}\right) q(A T(a) x),
\end{aligned}
$$

which implies that $T(\cdot) x$ is absolutely continuous on $[a, b]$.

The proof of the implication (ii) $\Rightarrow$ (iii) is entirely similar to that of the classical case and is omitted. To prove the implication (iii) $\Rightarrow$ (i) we shall need the following lemmas:

LemMa 1. Let $X$ be a reflexive locally convex linear topological space and $\{T(t): t \geqq 0\} \subset L(X)$ a one-parameter semigroup. If $\left\{y_{n}\right\}$ is a bounded sequence in $X$, then there exists $y \in X$ such that for each $x^{*} \in X^{*}$ there exists a subsequence $\left\{x_{n}\right\}$ of $\left\{y_{n}\right\}$ for which

$$
\lim _{n \rightarrow \infty}\left\langle T(t) x_{n}-T(t) y, x^{*}\right\rangle=0 \quad \text { for all } t \geqq 0 .
$$

Proof. Since $X$ is reflexive and $\left\{y_{n}\right\}$ is bounded, we may choose a weak cluster point $y \in X$ of $\left\{y_{n}\right\}$. Let $x^{*} \in X^{*}$ and let $r_{1}, r_{2}, \cdots$, be an enumeration of the nonnegative rationals. For each positive integer $n$ let

$$
U_{n}=\left\{x:\left|\left\langle y-x, T\left(r_{j}\right)^{*} x^{*}\right\rangle\right|\left\langle n^{-1}, 1 \leqq j \leqq n\right\},\right.
$$


where $T(t)^{*}$ denotes the operator dual of $T(t)$. Since $y$ is a weak cluster point of $\left\{y_{n}\right\}$, we may choose a subsequence $\left\{x_{n}\right\}$ of $\left\{y_{n}\right\}$ such that $x_{n} \in U_{n}$ for every $n$. Let $B$ be any bounded set in $X$ containing $\left\{y_{n}\right\}$. Then $q\left(y^{*}\right)=$ $\sup \left\{\left|\left\langle x, y^{*}\right\rangle\right|: x \in B\right\}$ defines a continuous seminorm for the strong topology of $X^{*}$, so by the reflexivity of $X$ it follows that (see [2])

$$
\lim _{r \rightarrow t} q\left(T(r)^{*} x^{*}-T(t)^{*} x^{*}\right)=0 .
$$

Let $\varepsilon>0, t \geqq 0$, and choose $r_{j}$ so that

$$
\sup _{n}\left|\left\langle T\left(r_{j}\right) x_{n}-T(t) x_{n}, x^{*}\right\rangle\right| \leqq q\left(T\left(r_{j}\right)^{*} x^{*}-T(t)^{*} x^{*}\right)<\varepsilon
$$

and

$$
\left|\left\langle T\left(r_{j}\right) y-T(t) y, x^{*}\right\rangle\right|<\varepsilon .
$$

If $n>\max \{j, 1 / \varepsilon\}$, then

and therefore

$$
\left|\left\langle x_{n}-y, T\left(r_{j}\right)^{*} x^{*}\right\rangle\right|<1 / n<\varepsilon,
$$

$$
\begin{aligned}
\left|\left\langle T(t) x_{n}-T(t) y, x^{*}\right\rangle\right| \leqq & \left|\left\langle T(t) x_{n}-T\left(r_{j}\right) x_{n}, x^{*}\right\rangle\right|+\left|\left\langle T\left(r_{j}\right)\left(x_{n}-y\right), x^{*}\right\rangle\right| \\
& +\left|\left\langle T\left(r_{j}\right) y-T(t) y, x^{*}\right\rangle\right|<3 \varepsilon,
\end{aligned}
$$

proving the lemma. Q.E.D.

Lemma 2. Let $X$ be a reflexive locally convex linear topological space and $\{T(t): t \geqq 0\} \subset L(X)$ a one-parameter semigroup with infinitesimal generator $A$. Then $x \in D(A)$ if and only if $\{(T(h) x-x) / h: 0<h<1\}$ is a bounded subset of $X$.

Proof. The necessity is obvious. To prove the sufficiency we note that by Lemma 1 we may choose a point $y$ in $X$ such that given any $x^{*} \in X^{*}$, there exists a sequence

$$
x_{n}=\left(T\left(h_{n}\right) x-x\right) / h_{n}, \quad h_{n} \searrow 0,
$$

such that for all $t \geqq 0$,

$$
\lim _{n \rightarrow \infty}\left\langle T(t) x_{n}, x^{*}\right\rangle=\left\langle T(t) y, x^{*}\right\rangle .
$$

Since $\{T(t): t \geqq 0\}$ is locally equicontinuous, given any positive $r$ there exists a continuous seminorm $p$ on $X$ such that for all $z \in X$ and all $0 \leqq t \leqq r,\left|\left\langle T(t) z, x^{*}\right\rangle\right| \leqq p(z)$. Therefore, since $\left\{x_{n}\right\}$ is bounded,

$$
\sup \left\{\left|\left\langle T(t) x_{n}, x^{*}\right\rangle\right|: 0 \leqq t \leqq r ; n=1,2, \cdots\right\}<\infty .
$$

By Lebesgue's dominated convergence theorem then,

$$
\lim _{n \rightarrow \infty} \int_{0}^{r}\left\langle T(t) x_{n}, x^{*}\right\rangle d t=\int_{0}^{r}\left\langle T(t) y, x^{*}\right\rangle d t .
$$


Since

$$
\int_{0}^{r} T(t) x_{n} d t=\left(1 / h_{n}\right) \int_{0}^{h_{n}}(T(t+r) x-T(t) x) d t \rightarrow T(r) x-x,
$$

it follows that

$$
\left\langle T(r) x-x, x^{*}\right\rangle=\left\langle\int_{0}^{r} T(t) y d t, x^{*}\right\rangle .
$$

Therefore, since $x^{*}$ was arbitrary,

$$
T(r) x-x=\int_{0}^{r} T(t) y d t,
$$

which implies that $x \in D(A)$. Q.E.D.

Lemma 3. Let $X$ be a locally convex linear topological space and $\{T(t)$ : $t \geqq 0\} \subset L(X)$ a locally equicontinuous one-parameter semigroup. Then for each continuous seminorm $p$ on $X$ and $0 \leqq a<b<\infty$ there exists a continuous seminorm $q$ on $X$ such that for all $x \in X$ and all $0<h<b-a$,

$$
p(T(b+h) x-T(b) x) / h \leqq L(x ; a, b ; q) /(b-a-h) .
$$

Proof. Let $p, a, b$ be given as in statement of lemma, and let $q$ be a continuous seminorm on $X$ such that $p(T(t) x) \leqq q(x)$ for all $x \in X$ and all $0 \leqq t \leqq b$. If $0<h<b-a$ and $n$ denotes the greatest integer in $(b-a) / h$, then for any integer $j$ such that $1 \leqq j \leqq n$ we have $0 \leqq b-a-j h+h \leqq b$, so

$$
\begin{aligned}
p(T(b+h) x- & T(b) x) \\
& =p(T(b-a-j h+h)(T(a+j h) x-T(a+j h-h) x)) \\
& \leqq q(T(a+j h) x-T(a+j h-h) x) .
\end{aligned}
$$

Therefore,

$$
\begin{aligned}
n p(T(b+h) x-T(b) x) & \leqq \sum_{j=1}^{n} q(T(a+j h) x-T(a+j h-h) x) \\
& \leqq L(x ; a, b ; q),
\end{aligned}
$$

and since $(b-a-h) / h<n$, the conclusion readily follows. Q.E.D.

Returning to the proof of the Theorem, let us assume that statement (iii) holds. Given any continuous seminorm $s$ on $X$, there exists, by virtue of the local equicontinuity of $T(t)$ and the fact that $T(c)$ is an isomorphism, a continuous seminorm $p$ such that $s(y) \leqq p(T(t) y)$ for all $y \in X$ and all $t \in[0, c]$. Hence, given any interval $[a, b] \subset[0, c]$ we obtain by Lemma 3 a continuous seminorm $q$ such that

$$
s(T(h) x-x) \cdot h^{-1} \leqq 2 L(x ; a, b ; q) \cdot(b-a)^{-1}
$$


for all $0<h<(b-a) / 2$. Therefore $\left\{h^{-1}(T(h) x-x): 0<h<1\right\}$ is a bounded set in $X$, hence $x \in D(A)$ by Lemma 2 .

To prove the final assertion of the Theorem, assume conditions (i)-(iii) hold and let $0 \leqq a<b<\infty$. Let $p$ be a continuous seminorm on $X$ and choose a sequence of sums

$$
S_{m}=\sum_{j=1}^{n(m)} p\left(T\left(t_{j m}\right) x-T\left(t_{j-1}\right) x\right),
$$

where $a=t_{0 m}<t_{1 m}<\cdots<t_{n(m) m}=b$, such that

$$
\lim _{m \rightarrow \infty} S_{m}=L(x ; a, b ; p) \text { and } \lim _{m \rightarrow \infty} \max _{j}\left(t_{j m}-t_{j-1 m}\right)=0 .
$$

Define $f_{m}:[a, b] \rightarrow[0, \infty)$ by

$$
f_{m}=\sum_{j=1}^{n(m)} p\left(\left(T\left(t_{j m}\right) x-T\left(t_{j-1}\right) x\right) /\left(t_{j m}-t_{j-1 m}\right)\right) \cdot \chi_{\left[t_{j-1} \cdot t_{j m}\right]}
$$

where $\chi_{Y}$ denotes the characteristic function of a set $Y \subset[a, b]$. If $t \in[a, b]$ is not equal to any partition point $t_{j m}$, then a standard calculation shows that $f_{m}(t) \rightarrow p(A T(t) x)$. Therefore $f_{m} \rightarrow p(A T(\cdot) x)$ a.e. in $[a, b]$. Also, the sequence $\left\{f_{m}\right\}$ is uniformly bounded a.e. For by the local equicontinuity of the semigroup there exists a continuous seminorm $q$ on $X$ such that if $t_{j-1 m}<t<t_{j m}$

$$
\begin{aligned}
f_{m}(t) & =\left(t_{j m}-t_{j-1 m}\right)^{-1} p\left(T\left(t_{j m}\right) x-T\left(t_{j-1}\right) x\right) \\
& \leqq\left(t_{j m}-t_{j-1 m}\right)^{-1} \int_{t_{j-1} m}^{t_{j m}} p(A T(s) x) d s \\
& \leqq q(A T(a) x) .
\end{aligned}
$$

Therefore, by Lebesgue's dominated convergence theorem,

$$
\int_{a}^{b} p(A T(t) x) d t=\lim _{m \rightarrow \infty} \int_{a}^{b} f_{m}(t) d t=\lim _{m \rightarrow \infty} S_{m}=L(x ; a, b ; p),
$$

completing the proof of the Theorem.

3. Proof of corollary. We observe first that given a continuous seminorm $q$ on $X$ and an interval $[a, b] \subset[0, \infty)$, there exists for each integer $n \geqq 1$ a continuous seminorm $p$ such that

$$
L(x ; a, b ; q) \leqq L(x ; a+n c, b+n c ; p) .
$$

Hence it suffices to prove the Corollary for the case $[a, b] \subset[0, c]$.

Let $U$ be a nonempty open subset of $X$. Since $D(A) \neq X$, there exists a point $x$ in $U \backslash D(A)$. By the proof of the Theorem, there exists a continuous 
seminorm $q$ such that $L(x ; a, b ; q)=+\infty$. Now define

$$
x(r)=r^{-1} \int_{0}^{r} T(t) x d t \quad(r>0) .
$$

Then $x(r) \in D(A) \cap U$ for all sufficiently small $r$, and $A x(r)=r^{-1}(T(r) x-x)$. By Lemma 2, there exists a continuous seminorm $s$ such that $s(A x(r))$ is arbitrarily large for small $r$. Choosing a continuous seminorm $p$ such that $s(A x(r)) \leqq p\left(T_{t} A x(r)\right)(0 \leqq t \leqq c)$ and noting that

$$
L(x(r) ; a, b ; p)=\int_{a}^{b} p(T(t) A x(r)) d t \geqq(b-a) s(A x(r)),
$$

we see that $L(x(r) ; a, b ; p)$ is arbitrarily large for small $r$.

\section{REFERENCES}

1. P. L. Butzer and H. Berens, Semi-groups of operators and approximation, Die Grundlehren der math. Wissenschaften, Band 145, Springer-Verlag, New York, 1967. MR 37 \#5588.

2. T. Kōmura, Semigroups of operators in locally convex spaces, J. Functional Analysis 2 (1968), 258-296. MR 38 \#2634.

Department of Mathematics, George Washington University, Washington, D.C. 20006 